\title{
Conformal Phase Transition and Fate of the Hidden Local Symmetry in Large $N_{f}$ QCD
}

\author{
Masayasu Harada and Koichi Yamawaki \\ Department of Physics, Nagoya University, Nagoya 464-8602, Japan.
}

\begin{abstract}
It is observed that the Hidden Local Symmetry (HLS) for the vector mesons in the ordinary QCD with smaller $N_{f}$ plays the role of the "Higgsed magnetic gauge symmetry" for the Seiberg duality in the SUSY QCD. For large $N_{f}$ where the conformal phase transition with chiral restoration and deconfinement is expected to take place, we find that the HLS model also exhibits the chiral restoration by the loop corrections (including the quadratic divergence) in a manner similar to that in the $C P^{N-1}$ model, provided that the bare HLS Lagrangian respects the Georgi's vector limit at a certain $N_{f}(\approx 7)$.
\end{abstract}

Increasing attention has been paid to the duality in various contexts of modern particle theory. Seiberg found the "electric-magnetic" duality in $\mathcal{N}=1$ Supersymmetric (SUSY) QCD with $N_{c}$ colors and $N_{f}$ flavors [1]: For the region $\frac{3}{2} N_{c}<N_{f}<3 N_{c}$ ("conformal window") in the SUSY QCD, there exists a "magnetic theory" with the $\mathrm{SU}\left(N_{f}-N_{c}\right)$ gauge symmetry which is dual to the original $\mathrm{SU}\left(N_{c}\right)$ theory regarded as the "electric theory". Although the origin of the magnetic gauge symmetry ("induced at the composite level") is not obvious from the original theory, both theories in fact have the infrared (IR) fixed point with exact conformal symmetry and with the same IR physics. When $N_{f}$ decreases, the electric theory becomes stronger in IR, while the magnetic theory gets weaker, with the magnetic gauge group being reduced through the Higgs mechanism. Decreasing $N_{f}$ further beyond the conformal window, we finally arrive at $N_{f}=N_{c}$ where the magnetic theory is in complete Higgs phase (reduced to no gauge group), which corresponds to the complete confinement (and spontaneously broken chiral symmetry) of the electric theory.

Similar conformal window may also exist in the ordinary (non-SUSY) QCD with massless $N_{f}$ flavors. There actually exists an IR fixed point at two-loop beta function for large $N_{f}\left(<\frac{11}{2} N_{c}\right)$ : When $N_{f}$ increases close to the point $\frac{11}{2} N_{c}$, the coupling at the IR fixed point becomes very small so that the deconfinement and the chiral symmetry restoration are expected to occur [2]. Based on this IR fixed point, it was found [3] through the modified ladder Schwinger-Dyson (SD) equation that chiral symmetry restoration in fact takes place for $N_{f}$ such that $N_{f}^{\mathrm{cr}}<N_{f}<\frac{11}{2} N_{c}$, where $N_{f}^{\mathrm{cr}} \simeq 4 N_{c}\left(=12\right.$ for $\left.N_{c}=3\right)$. In Ref. [4 this chiral restoration at $N_{f}^{\text {cr }}$ was further identified with "conformal phase transition" which was characterized by the essential singularity scaling. Moreover, the lattice simulation indicates that the chiral restoration does occur at $N_{f}^{\mathrm{cr}} \approx 7$ [5].

Here we recall that, for small $N_{f}$, the vector mesons such as the $\rho$ meson can be regarded as the dynamical gauge bosons of Hidden Local Symmetry (HLS) [6, ] ] in the nonlinear sigma model (Chiral Lagrangian). The HLS is completely broken through the Higgs mechanism as the origin of the vector meson mass. This gauge symmetry is induced at the composite level and has nothing to do with the fundamental color gauge symmetry. Instead, the HLS is associated with the flavor symmetry.

In this paper we shall find that the Seiberg duality is realized also in the ordinary (non-SUSY) QCD through the HLS. This will shed new light on the non-perturbative dynamics of the real-life QCD.

We first observe that, for small $N_{f}$, the $\mathrm{SU}\left(N_{f}\right)$ HLS is in complete Higgs phase and yields the same IR physics as the $\mathrm{SU}\left(N_{c}\right)$ QCD in the confinement/chiral-symmetrybreaking phase, and plays the role of the "Higgsed magnetic gauge theory" dual to the "confined electric gauge theory" (QCD) in the spirit of Seiberg duality.

What then happens to the HLS when $N_{f}$ becomes large so that QCD undergoes the conformal phase transition into the conformal window with deconfinement/chiral restoration? In order for the duality between QCD and the HLS be consistently satisfied, there should be a way that the chiral restoration takes place for large $N_{f}$ also in the HLS theory by its own dynamics. Actually, it is known that, in the $C P^{N-1}$ nonlinear sigma model based on the coset space $\mathrm{SU}(N) / \mathrm{SU}(N-1) \times \mathrm{U}(1)$, the $\mathrm{SU}(N)$ symmetry is restored by the loop effects and the U(1) gauge symmetry is dynamically generated accordingly (See, e.g., Ref. [7].). This suggests that the HLS can provide the chiral restoration by its own dynamics. In other words, due to the dynamics of the HLS the quantum theory is in the symmetric phase even if the bare theory is written as if it were in the broken phase.

Now our task is to find a condition for the bare theory of the HLS to realize chiral restoration for large $N_{f}$ in the quantum theory. One clue is the fact [8] that through the renormalization-group equations (RGE's) the HLS approaches to the Georgi's vector limit [9] in the idealized high energy limit. The vector limit is actually the ultraviolet fixed point of the RGE's.

We then propose that taking the Georgi's vector limit [9] in the bare theory of the HLS at a certain critical value $N_{f}^{c r}$ is a consistent way to incorporate the conformal phase transition into the HLS. In other words, the quantum theory provides the chiral restoration when the 
bare theory approaches to the vector limit as $N_{f} \rightarrow N_{f}^{c r}$.

Let us first describe the HLS model based on the $G_{\text {global }} \times H_{\text {local }}$ symmetry, where $G=\mathrm{SU}\left(N_{f}\right)_{L} \times$ $\mathrm{SU}\left(N_{f}\right)_{R}$ is the global chiral symmetry and $H=$ $\mathrm{SU}\left(N_{f}\right)_{V}$ is the HLS. (The flavor symmetry is the diagonal sum of $G_{\text {global }}$ and $H_{\text {local }}$.) The Lagrangian is [6,7]

$$
\mathcal{L}=F_{\pi}^{2} \operatorname{tr}\left[\hat{\alpha}_{\perp \mu} \hat{\alpha}_{\perp}^{\mu}\right]+F_{\sigma}^{2} \operatorname{tr}\left[\hat{\alpha}_{\| \mu} \hat{\alpha}_{\|}^{\mu}\right]+\mathcal{L}_{\text {kin }}\left(\rho_{\mu}\right),
$$

where $\mathcal{L}_{\text {kin }}\left(\rho_{\mu}\right)$ denotes the kinetic term of the gauge boson $\rho_{\mu}$ of the HLS (vector meson) and

$$
\hat{\alpha}_{\perp}^{\mu}=\left(D^{\mu} \xi_{\mathrm{L}} \cdot \xi_{\mathrm{L}}^{\dagger} \mp D^{\mu} \xi_{\mathrm{R}} \cdot \xi_{\mathrm{R}}^{\dagger}\right) /(2 i) .
$$

Two $\mathrm{SU}\left(N_{f}\right)$-matrix valued variables $\xi_{\mathrm{L}}$ and $\xi_{\mathrm{R}}$ transform as $\xi_{\mathrm{L}, \mathrm{R}}(x) \rightarrow \xi_{\mathrm{L}, \mathrm{R}}^{\prime}(x)=h(x) \xi_{\mathrm{L}, \mathrm{R}}(x) g_{\mathrm{L}, \mathrm{R}}^{\dagger}$, where $h(x) \in H_{\text {local }}$ and $g_{\mathrm{L}, \mathrm{R}} \in G_{\text {global }}$. These variables are parameterized as $\xi_{\mathrm{L}, \mathrm{R}}=e^{i \sigma / F_{\sigma}} e^{\mp i \pi / F_{\pi}}$, where $\pi=\pi^{a} T_{a}$ denotes the Nambu-Goldstone (NG) boson associated with the spontaneous breaking of $G$ chiral symmetry and $\sigma=\sigma^{a} T_{a}$ denotes the NG boson absorbed into the HLS gauge boson. $F_{\pi}$ and $F_{\sigma}$ are relevant decay constants, and the parameter $a$ is defined as $a \equiv F_{\sigma}^{2} / F_{\pi}^{2}$. The covariant derivatives of $\xi_{\mathrm{L}, \mathrm{R}}$ are defined by $D_{\mu} \xi_{\mathrm{L}, \mathrm{R}}=$ $\partial_{\mu} \xi_{\mathrm{L}, \mathrm{R}}-i g \rho_{\mu} \xi_{\mathrm{L}, \mathrm{R}}$, where $g$ is the HLS gauge coupling. In this paper we use $\pi$ for the pseudoscalar NG bosons associated with the $G$ breaking and $\rho$ for the HLS gauge bosons even for $N_{f} \neq 2$.

We adopt the background gauge field method to obtain quantum corrections to the parameters. This method was used in the chiral perturbation theory (ChPT) [10, and was applied to the HLS in Ref. 11. We define two decay constants $F_{\pi}$ and $F_{\sigma}$ from two-point functions of the background fields $\hat{\alpha}_{\perp}^{\mu}$ and $\hat{\alpha}_{\|}^{\mu}$, respectively. The HLS gauge coupling $g$ is determined from two-point function of the gauge field strength. We note that the would-be NG boson $\sigma$ does contribute in the $R_{\xi}$-like gauge fixing [8] or the background gauge fixing 11 .

To handle the quantum effects properly and relate parameters in the bare theory defined at the cutoff scale with those in the quantum theory at lower energy scale, we use the RGE's in the Wilsonian sense which include the quadratic divergences in addition to the logarithmic divergences. As is usual, inclusion of only the logarithmic divergences is not adequate to study the phase structure. Actually, in the above $C P^{N-1}$ model in $D(2<D<4)$ dimensions it was essential to include the power divergence which is the quadratic divergence in 4 dimensions. As we shall show, the vector limit is still the fixed point even if we include the quadratic divergences.

The RGE's for $g$ and $a$ above the $\rho$ mass scale with only the logarithmic divergences are shown in Ref. [8] where the parameters renormalized in the mass independent scheme were studied and the vector limit was shown to be realized in the high energy limit. Here we further include the quadratic divergences, since we need RGE's in the Wilsonian sense to study the phase structure. Since a naive momentum cutoff violates the chiral symmetry, we need a careful treatment of the quadratic divergences. Thus we adopt the dimensional regularization and identify the quadratic divergences with the presence of poles of ultraviolet origin at $n=2[12]$. We show the diagrams contributing to two-point functions of $\hat{\alpha}_{\perp}^{\mu}$ and $\hat{\alpha}_{\|}^{\mu}$ in Figs. 1 and 2 [13]. The resultant RGE's above the $\rho$ mass scale are given by

$$
\begin{aligned}
\mu \frac{d F_{\pi}^{2}}{d \mu} & =C\left[3 a^{2} g^{2} F_{\pi}^{2}+2(2-a) \mu^{2}\right], \\
\mu \frac{d g^{2}}{d \mu} & =-C \frac{87-a^{2}}{6} g^{4}, \\
\mu \frac{d a}{d \mu} & =-C(a-1)\left[3 a(a+1) g^{2}-(3 a-1) \frac{\mu^{2}}{F_{\pi}^{2}}\right],
\end{aligned}
$$

where $C=N_{f} /\left(2(4 \pi)^{2}\right)$ and $\mu$ is the renormalization scale. The first term of Eq. (3) comes from the logarithmic divergences of the diagrams in Figs. 11(a) and (b), while the second term comes from the quadratic divergences of the diagrams in Figs. 目(b) and (c). The RGE for $a$ is obtained from those for $F_{\pi}$ and $F_{\sigma}$ through the definition $a \equiv F_{\sigma}^{2} / F_{\pi}^{2}$. We note here that the above RGE's agree with those obtained in Ref. [8] when we neglect the quadratic divergences. As is easily read from the RGE's (画) and (5) the Georgi's vector limit [9] $(g, a)=$ $(0,1)$ is the fixed point. The mass of $\rho$ is determined by the on-shell condition: $m_{\rho}^{2}=a\left(m_{\rho}\right) g^{2}\left(m_{\rho}\right) F_{\pi}^{2}\left(m_{\rho}\right)$.

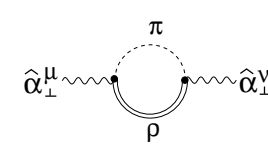

(a)

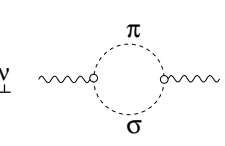

(b)

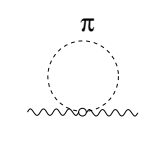

(c)
FIG. 1. Diagrams contributing to two-point function of $\hat{\alpha}_{\perp}^{\mu}$.

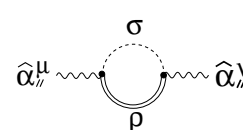

(a)

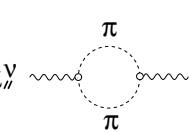

(b)

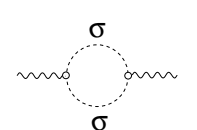

(c)

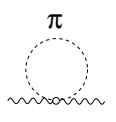

(d)
FIG. 2. Diagrams contributing to two-point function of $\hat{\alpha}_{\|}^{\mu}$.

Now, let us study how the quantum theory approaches to the chiral symmetric phase when $N_{f}$ becomes larger. Here and henceforth we write the dependence on $N_{f}$ as well as on the scale $\mu$ explicitly. $F_{\pi}$ in the bare theory can be identified with that at the cutoff scale in the Wilsonian renormalization scheme. This cutoff scale, say $\Lambda$, generally depends on $N_{f}$, so we express this by $\Lambda_{f} \equiv \Lambda\left(N_{f}\right)$. As we stated before, the bare theory is written as if it were in the broken phase. Then the parameter $F_{\pi}$ at the cutoff scale does not vanish, and it is natural to assume that $F_{\pi}\left(\Lambda_{f}\right)$ is of order of $\Lambda_{f}$ : 


$$
F_{\pi}\left(\Lambda_{f} ; N_{f}\right) \sim \Lambda_{f}
$$

Actually, the phase is determined by studying whether $F_{\pi}\left(0 ; N_{f}\right)$, which is the decay constant of NG bosons in the quantum theory, vanishes or not. The order parameter $F_{\pi}\left(0 ; N_{f}\right)$ will vanish due to the loop effects of $\rho$ and $\pi$ 14. This phenomena actually occurs if the bare theory approaches to the vector limit as $N_{f}$ becomes large. Since the vector limit is the fixed point, we may solve the RGE for $F_{\pi}$ Eq. (3) in the vector limit with taking $(g, a)=(0,1)$. This RGE tells us that $F_{\pi}$ does not diverge, and hence we conclude that $m_{\rho}=0$ in the vector limit. Thus the RGE (3) with $(g, a)=(0,1)$ relates the order parameter $F_{\pi}\left(0 ; N_{f}\right)$ with $F_{\pi}\left(\Lambda_{f} ; N_{f}\right)$ as 15

$$
\frac{F_{\pi}^{2}\left(m_{\rho}=0 ; N_{f}\right)}{\Lambda_{f}^{2}}=\frac{F_{\pi}^{2}\left(\Lambda_{f} ; N_{f}\right)}{\Lambda_{f}^{2}}-\frac{N_{f}}{2(4 \pi)^{2}} .
$$

Since $F_{\pi}\left(\Lambda_{f} ; N_{f}\right) \sim \Lambda_{f}$, right-hand side (RHS) of Eq. (7) will vanish for a large value of $N_{f}$. The chiral symmetry in the quantum theory is restored at a certain flavor $N_{f}^{\text {cr }}$ when we take the vector limit in the bare theory:

$$
F_{\pi}\left(0 ; N_{f}\right) / \Lambda_{f} \underset{N_{f} \rightarrow N_{f}^{\text {cr }}}{\longrightarrow} 0 .
$$

This is our main result.

Let us next calculate the critical flavor $N_{f}^{\text {cr }}$. Here we use the following "physical" inputs for $N_{f}=3$ with taking $a=1$ [16]: $F_{\pi}\left(0 ; N_{f}=3\right)=88 \mathrm{MeV}$ determined in the chiral limit 10; $m_{\rho}\left(N_{f}=3\right)=770 \mathrm{MeV}$; $\Lambda\left(N_{f}=3\right)=4 \pi F_{\pi}\left(0 ; N_{f}=3\right) \simeq 1.1 \mathrm{GeV}$ from the naive dimensional analysis [17]. Below the $m_{\rho}$ scale, $\rho$ decouples and hence $F_{\pi}$ runs by the loop effect of $\pi$ alone. The relevant Lagrangian with least derivatives is given by the first term of Eq. (11), and the diagram contributing to $F_{\pi}^{2}$ is shown in Fig. 1(c). The resultant RGE for $F_{\pi}$ is given by $\left(d F_{\pi}^{2}\right) /\left(d \mu^{2}\right)=2 C$. Then the order parameter $F_{\pi}\left(0 ; N_{f}\right)$ is related to $F_{\pi}\left(m_{\rho}\left(N_{f}\right) ; N_{f}\right)$ by

$$
F_{\pi}^{2}\left(0 ; N_{f}\right)=F_{\pi}^{2}\left(m_{\rho}\left(N_{f}\right) ; N_{f}\right)-\frac{N_{f}}{(4 \pi)^{2}} m_{\rho}^{2}\left(N_{f}\right) .
$$

The above input leads to $F_{\pi}\left(\Lambda_{3} ; N_{f}=3\right)=171 \mathrm{MeV}$ and $g\left(m_{\rho} ; N_{f}=3\right) \simeq 5.6$, the latter of which is consistent with the values of $g$ determined by assuming the saturation of the ChPT parameter $L_{9}\left(m_{\rho}\right)$ by the vector mesons [18]; $g\left(m_{\rho} ; N_{f}=3\right)=6.0 \pm 0.4$. For simplicity we assume that $\Lambda_{f}$ and $F_{\pi}\left(\Lambda_{f} ; N_{f}\right) / \Lambda_{f}$ do not depend on $N_{f} ; F_{\pi}^{2}\left(\Lambda_{f} ; N_{f}\right) / \Lambda_{f}^{2} \simeq 0.024$. Then the critical flavor is determined from Eq. (7) as

$$
N_{f}^{\mathrm{cr}} \simeq 7.6
$$

which is somewhat similar to the lattice calculation [5].

To study how $F_{\pi}$ approaches to zero as $N_{f}$ is increased we first need to determine how the bare parameters $g\left(\Lambda_{f} ; N_{f}\right)$ and $a\left(\Lambda_{f} ; N_{f}\right)$ approach to the values in the vector limit [19]. In the present analysis let us fix $a \equiv 1$ for simplicity [20]. We adopt the following behavior of the gauge coupling approaching to zero:

$$
g^{2}\left(\Lambda_{f} ; N_{f}\right)=\bar{g}^{2} \epsilon, \quad \epsilon \equiv 1 / N_{f}-1 / N_{f}^{\mathrm{cr}},
$$

where $\bar{g}$ is independent of $N_{f}$ [21]. We present numerical calculation of the $N_{f}$-dependence of $F_{\pi}\left(0 ; N_{f}\right)$ in Fig. 3. This clearly shows that $F_{\pi}\left(0 ; N_{f}\right)$ smoothly goes to zero [22] at $N_{f}^{\mathrm{cr}} \simeq 7.6$. Thus we conclude that the quantum theory provides the chiral restoration when the bare theory approaches to the vector limit as $N_{f} \rightarrow N_{f}^{c r}$.

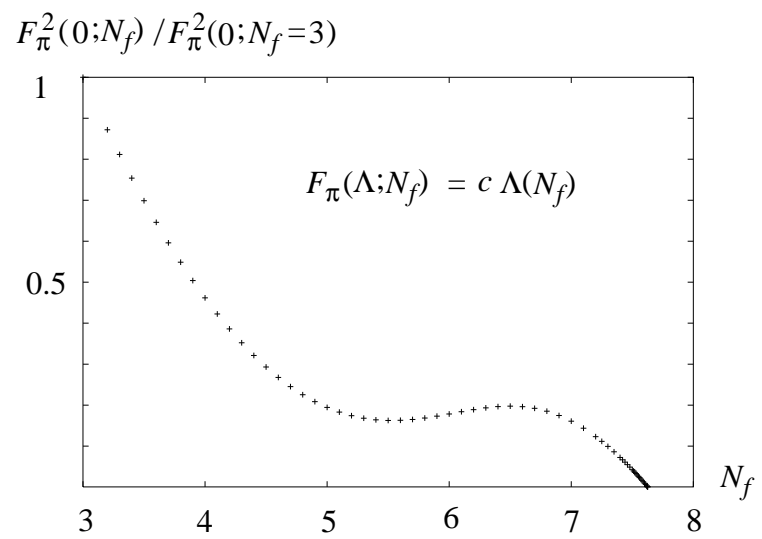

FIG. 3. $\quad N_{f}$-dependence of $F_{\pi}^{2}\left(0 ; N_{f}\right)$, normalized by $F_{\pi}^{2}\left(0 ; N_{f}=3\right)$. The constant $c \simeq 0.16$ is determined from the physical inputs for $N_{f}=3$ discussed in the text.

Several comments are in order:

In this paper we numerically studied the $N_{f^{-}}$ dependence of $F_{\pi}\left(0 ; N_{f}\right)$. However, the RGE's are analytically solvable when we take $a=1$, and the critical behaviors of $F_{\pi}\left(0 ; N_{f}\right)$ and $m_{\rho}\left(N_{f}\right)$ can be studied analytically. A careful analysis 23] of the solutions of RGE's with the condition (11) leads to the fact that when $N_{f} \rightarrow$ $N_{f}^{\mathrm{cr}}, g^{2}\left(m_{\rho}\left(N_{f}\right) ; N_{f}\right) \sim \epsilon, F_{\pi}^{2}\left(m_{\rho}\left(N_{f}\right) ; N_{f}\right) / \Lambda_{f}^{2} \sim \epsilon$, and hence $m_{\rho}^{2}\left(N_{f}\right) / \Lambda_{f}^{2} \sim \epsilon^{2}$. This implies that the second term of RHS of Eq. (9) approaches to zero faster than the first term. Thus we obtain the critical behavior of the order parameter as $F_{\pi}^{2}\left(0 ; N_{f}\right) / \Lambda_{f}^{2} \sim \epsilon$ 24]. This shows that the physical parameters $F_{\pi}(0)$ and $m_{\rho}$ approaches to zero in the power behavior, which is originated from the fact that we have used the one-loop perturbative RGE's. If, on the other hand, we use some non-perturbative treatment, we might obtain an essential singularity scaling shown by an analysis of the SD equation [3. 4 .

In the present analysis we took $F_{\pi}\left(\Lambda_{f}\right) / \Lambda_{f}$ as well as $\Lambda_{f}$ as a quantity independent of $N_{f}$. At the scale of $\Lambda_{f}$ we would like to match the HLS with QCD, so that $N_{f}$-dependence of $\Lambda_{f}$ may be extracted from QCD. However, as we can easily read from Eq. (7), imposing an $N_{f}$-dependence (increasing or decreasing) of $\Lambda_{f}$, with $F_{\pi}\left(\Lambda_{f}\right) / \Lambda_{f}$ fixed, does not change the critical flavor. On 
the other hand, if $F_{\pi}\left(\Lambda_{f}\right) / \Lambda_{f}$ depends on $N_{f}$, the critical flavor will be slightly changed. For example, we can include the effect of the anomalous dimension obtained from QCD by using the Pagels-Stokar formula for $F_{\pi}\left(\Lambda_{f}\right)$ integrated over the region $p^{2}>\Lambda_{f}^{2}$. This changes the resultant value of $N_{f}^{\text {cr }}$ to $N_{f}^{\text {cr }} \simeq 6.5$. A detailed analysis will be shown in the forthcoming paper [23].

Axialvector mesons such as $a_{1}$ are heavier than the cutoff scale, $\Lambda\left(N_{f}=3\right) \simeq 1.1 \mathrm{GeV}$, so that we did not include them here. On the other hand, the recent analyses [25] show that there exist light scalar mesons. Since the phase transition is expected to be the conformal phase transition, those masses as well as the baryon masses will be small anyway [26] when $N_{f}$ approaches to $N_{f}^{\text {cr }}$. Thus the critical flavor obtained in this paper $N_{f}^{\text {cr }} \simeq 7.6$ might be changed by including those effects.

Although we can study the phase transition only from the broken phase in this framework, it would be interesting to see whether the HLS still makes sense even in the conformal window.

Our result may be applied to the dynamical electroweak symmetry breaking such as technicolor. As we discussed above, the $\rho$ (technirho) mass near the critical point becomes much smaller than $F_{\pi}\left(0 ; N_{f}\right)$ which is fixed to be the weak scale. Thus we expect the light technirho as a signal in the future collider experiments.

[1] N. Seiberg, Nucl Phys. B435 129 (1995).

[2] T. Banks and A. Zaks, Nucl. Phys. B 196, 189 (1982).

[3] T. Appelquist, J. Terning and L.C.R. Wijewardhara, Phys. Rev. Lett. 77, 1214 (1996); T.W. Appelquist, A. Ratnaweera, J. Terning and L.C.R. Wijewardhana, Phys. Rev. D58, 105017 (1998).

[4] V.A. Miransky and K. Yamawaki, Phys. Rev. D 55, 5051 (1997).

[5] Y. Iwasaki, K. Kanaya, S. Kaya, S. Sakai and T. Yoshié, hep-lat/9804005; Nucl. Phys. B53 (Proc. Suppl.), 449 (1997).

[6] M. Bando, T. Kugo, S. Uehara, K. Yamawaki and T. Yanagida, Phys. Rev. Lett. 54, 1215 (1985).

[7] M. Bando, T. Kugo and K. Yamawaki, Phys. Rep. 164, 217 (1988).

[8] M. Harada and K. Yamawaki, Phys. Lett. B 297, 151 (1992).

[9] H. Georgi, Phys. Rev. Lett. 63, 1917 (1989); Nucl. Phys. B 331, 311 (1990).

[10] J. Gasser and H. Leutwyler, Ann. Phys. (N.Y.) 158, 142 (1984); Nucl. Phys. B250, 465 (1985).

[11] M. Tanabashi, Phys. Lett. B 316, 534 (1993).

[12] M. Veltman, Acta. Phys. Polon. B12, 437 (1981)

[13] M. Harada and A. Shibata, Phys. Rev. D 55, 6716 (1997).

[14] If we neglected the contributions from $\rho$, the relation between $F_{\pi}\left(\Lambda_{f} ; N_{f}\right)$ and $F_{\pi}\left(0 ; N_{f}\right)$ would be obtained by replacing $m_{\rho}\left(N_{f}\right)$ with $\Lambda_{f}$ in Eq. (9). Then the chiral restoration would have occurred for $N_{f} \sim 4$. However, the vector meson is in fact lighter than the cutoff scale already for $N_{f}=3$, and hence we cannot neglect the contributions from $\rho$.

[15] Here $\sigma$ does contribute even for $(g, a)=(0,1)$, since $\sigma$ is the real NG boson. Thus there is an extra factor $1 / 2$ in the second term of Eq. (7) compared with that of Eq. (9).

[16] In this paper the parameters are defined in the Wilsonian sense, and we study the parameter $a$ only above the $\rho$ mass scale $m_{\rho}$. The vector meson dominance of the pion form factor, which leads to $a=2$, was argued in the low energy region. In the presence of the quadratic divergence, however, the value of the parameter $a$ above the scale of $m_{\rho}$ differs from 2 . To give a quantitative feeling let us naively extend the present RGE for $a$ below $m_{\rho}$ by including only the $\pi$ effect shown in Fig. 2(d). Then we obtain $a\left(0 ; N_{f}=3\right) \simeq 1.8$ even for $a\left(m_{\rho} ; N_{f}=3\right)=1$.

[17] A. Manohar and H. Georgi, Nucl. Phys. B234, 189 (1984).

[18] G. Ecker, J. Gasser, A. Pich and E. De Rafael, Nucl. Phys. B321, 311 (1989).

[19] Here we impose that the bare theory approaches to the vector limit at $N_{f}^{\text {cr }}$, although it would be possible that the vector limit was already realized at $N_{f}<N_{f}^{\text {cr }}$.

[20] When we include a deviation of $a\left(m_{\rho} ; N_{f}=3\right)$ from one, the parameter $a$ runs by the RGE (5). However, the value of the critical flavor as well as the qualitative structure changes only slightly. (See Ref. 23.)

[21] This behavior may be justified by the following large $N_{f}$ argument. $g$ is the coupling of the three-point interaction of the vector mesons. Then large $N_{c}$ argument of QCD tells us that $g^{2}$ behaves as $1 / N_{c}$ in the large $N_{c}$ limit with fixed $N_{f}$. On the other hand, to make a large $N_{f}$ expansion in the HLS consistent the gauge coupling $g^{2}$ falls as $1 / N_{f}$. However, the HLS is actually related to QCD, so that the large $N_{f}$ limit should be taken with $N_{c} / N_{f}$ finite. This situation can be seen by rewriting the RHS of Eq. (11) into $\left(\bar{g}^{2} / N_{c}\right)\left(N_{c} / N_{f}-N_{c} / N_{f}^{\mathrm{cr}}\right)$.

[22] There is a small dip around $N_{f}=5$. As we stated in [14, below the $m_{\rho}$ scale $F_{\pi}$ decreases rapidly as $\mu$ decreases with fixed $N_{f}$ by the $\pi$ loop effect. This effect becomes larger as $N_{f}$ increases. Thus the chiral symmetry might have been restored by the effect. However, for $N_{f} \gtrsim 5$ the $\rho$ mass becomes small rapidly enough to protect such a situation to happen.

[23] M. Harada and K. Yamawaki, in preparation.

[24] When we change the power of $\epsilon$ in Eq. (11) from one to $q$ $(q>1), m_{\rho}\left(N_{f}\right)$ approaches to zero as $m_{\rho}^{2}\left(N_{f}\right) / \Lambda_{f}^{2} \sim$ $\epsilon^{1+q}$, while the critical behavior of $F_{\pi}\left(0 ; N_{f}\right)$ is not changed: $F_{\pi}^{2}\left(0 ; N_{f}\right) / \Lambda_{f}^{2} \sim \epsilon$. (See Ref. 23.)

[25] See, e.g., M. Harada, F. Sannino and J. Schechter, Phys. Rev. D 54, 1991 (1996); Phys. Rev. Lett. 78, 1603 (1997): N.A. Törnqvist and M. Roos, Phys. Rev. Lett. 76, 1575 (1996): S. Ishida, M.Y. Ishida, H. Takahashi, T. Ishida, K. Takamatsu and T. Tsuru, Prog. Theor. Phys. 95, 745 (1996): D. Morgan and M. Pennington, Phys. Rev. D 48, 1185 (1993): G. Janssen, B.C. Pearce, K. Holinde and J. Speth, Phys. Rev. D 52, 2690 (1995).

[26] R.S. Chivukula, Phys. Rev. D 55, 5238 (1997). 